\title{
ARTICLE
}

\section{Use of seclusion for managing behavioural disturbance in patients}

\author{
Giles Newton-Howes
}

Giles Newton-Howes is a consultant psychiatrist with the regional personality disorder service in the Lower North Island, New Zealand. His research interests include the interface between psychiatry and society, personality disorders and the impact of personality in mental state disorders.

Correspondence Dr Giles Newton-Howes, Department of Psychological Medicine, Private Bag 9014, University of Otago, Wellington 4156, New Zealand. Email: giles.newton-howes@otago. ac.nz

\begin{abstract}
SUMMARY
Seclusion is a tool used by psychiatrists primarily to manage aggressive and disturbed behaviour that is presumed to be due to the patient's mental disorder. In most parts of the world there are guidelines to using seclusion that are designed to maximise a patient's freedoms and protect their liberty while providing a safe environment. Arguments against the use of seclusion revolve around the deprivation of liberty, the potential for misuse and the concept of seclusion as a form of social control, and patients generally report seclusion as a negative, coercive experience. There is little evidence that seclusion provides long-term benefits in terms of treating symptoms or reducing aggression, although the literature is mixed in this regard. Expert opinion recommends a combination of national policy, ward management and patient-centred interventions to reduce seclusion rates.
\end{abstract}

\section{DECLARATION OF INTEREST}

None.

The management of aggression, violence and behavioural disturbance remains a challenging problem for psychiatric services despite advances in pharmacotherapy, psychological interventions for many mental disorders and improvement in the delivery of community psychiatric services. Notwithstanding the continuing trend of delivering psychiatric care in the community with the active input of patients and their families (Sowers 2005), the need for hospital-based management of acutely unwell patients remains. Hospital care is provided to the most unwell or highest-risk patients, and, as part of this in-patient care, seclusion and restraint are used to ensure patient safety and facilitate overall recovery.

\section{Historical considerations}

The use of seclusion and restraint has a long history, with its origin in the inhumane treatment of patients with psychiatric disorders in the 18th century and earlier. During these times, patients were often locked in foul and unclean rooms with little light and/or held in manacles. Pussin and
Pinel are credited in 1793 with the implementation of 'traitement moral', the dramatic improvement in the environmental conditions for the incurably insane at the Bicêtre asylum in France. This revolution did not occur in isolation, with similar improvements championed by Chiarugi in Italy and the Quaker William Tuke in England. In 1796, Tuke established The Retreat in northern England, where the use of manacles and chains - common practice in asylums at that time - was banned (as was physical punishment). The Retreat continues to operate today as a private hospital for patients in mental distress, with the core values of hope, courage, tolerance and valuing everyone. Although the practices of the 18th century are distant memories, the use of seclusion and restraint continues to be scrutinised in part on the basis of this history.

More recently, the trend in psychiatry is towards a more patient-focused, communitycentred approach, with decreasing emphasis on hospital-based care. This process, started by deinstitutionalisation, has led to a gradual reduction in hospital bed numbers over the past three decades. Consequently, an increasing specialisation is occurring in hospitals managing patients with acute mental disorder and associated behavioural disturbance. This means the proportion of 'difficult-to-manage behaviours' in in-patient settings remains high, as does the need to provide safe and appropriate care. In this context, seclusion remains an important clinical tool, although its history reminds us of the need for cautious use.

\section{What is seclusion?}

Although the term seclusion may be selfexplanatory to those who work in psychiatric settings, it is poorly defined. It largely depends on who is describing seclusion, i.e. medical bodies, policy makers, researchers or legal sectors. For example, the Royal College of Psychiatrists defines seclusion as:

'the supervised confinement of a patient specifically placed alone in a locked room for a period at any time of the day or night for the protection of the patient, staff or others from serious harm. The 
containment of a patient in a room such as a seclusion room when the door is not locked does not count as seclusion' (Public Policy Committee 1990).

More recently in England and Wales, following the legal changes brought about by a revision of the Mental Health Act 1983, seclusion is now defined by the Code of Practice as:

\begin{abstract}
'the supervised confinement of a patient in a room, which may be locked. Its sole aim is to contain severely disturbed behaviour which is likely to cause harm to others.

Alternative terminology such as "therapeutic isolation", "single-person wards" and "enforced segregation" should not be used to deprive patients of the safeguards established for the use of seclusion [Italics added] (Department of Health 2008: pp. 122-123)
\end{abstract}

This policy document has become the practical standard against which services are audited.

In Australia, the legal definition of seclusion is:

'the sole confinement of a person at any hour of the day or night in a room of which the doors and windows are locked from the outside' (Mental Health Act 1986: section 82(1)).

The unifying feature of the descriptions of seclusion is the confinement of a patient, separate from other patients. Although the above descriptions make this clear, separation can be defined broadly (as being in a locked ward) or narrowly (such as being locked in a room). Historically, the notion of 'time-out' would not have been considered seclusion, rather an operant conditioning technique to discourage inappropriate behaviours, although this would clearly fall under the modern rubric. Further obscuring the definition of seclusion is the admixture of the terms seclusion and restraint, with many policy documents and most research using both terms loosely.

From a pragmatic perspective, seclusion is best defined as the isolation of a patient from others for the purpose of managing behavioural disturbance. This is usually in a room that the patient cannot leave and in which they can be monitored continuously. The room itself is commonly 'low stimulus' or spartan in its furnishings, with few (if any) loose items that can be used in a dangerous manner by the patient. It is not generally considered to be a treatment per se, rather a necessary form of containment in order to minimise the harm a patient can do to themselves and to others (Box 1).

\section{The ethical and legal framework}

Despite the fact that seclusion remains mainstream in clinical practice internationally (Mason 1994) and is sanctioned in international law (United Nations 1991), its use remains controversial,
BOX 1 Key descriptors of seclusion

- Locked room

- Isolated from other patients and staff

- Spartan in its furnishings

- Few (or no) loose items

- The patient cannot leave the room

- The patient can be continuously observed

being described as social control (Morrall 2002) and felt as manipulative and punitive by patients (Holmes, 2004). There is no doubt that seclusion is coercive, except in the rare instance where it is requested by the patient, usually in the form of an advanced directive. Even in this case it may be felt as coercive at the time of implementation by the patient. From a bioethical perspective, seclusion reduces a patient's autonomy both in terms of restricting their physical freedoms and in increasing the likelihood of being forcibly medicated. The prima facie likelihood causing harm to the patient explains the risk management strategies used to minimise any legal exposure for clinicians involved in its use (Knox 2012). This runs in direct contradiction to the movement towards increased patient autonomy and choice more widely in psychiatric practice, taking a 'least restrictive' approach and recognising the importance of allowing patients to guide their own recovery (Davidson 2005). Bearing all this in mind, it may seem somewhat surprising that seclusion remains ethically justified as a part of everyday clinical practice.

Balancing this is the duty of care mental health staff have towards their patients and the recognition that patients who are sufficiently disturbed to require seclusion generally lack the capacity to make informed decisions about their care. Patients also place themselves at risk of accidental self-harm and are vulnerable to retribution for behaviours presumed to be driven by mental disorder. Similarly, mental health staff have a broader duty of care to all the patients in an in-patient setting, ensuring that patients do not harm each other. The consequences of violence are obviously detrimental to a victim, but also to a perpetrator. The loss of autonomy due to mental disorder, inability to give informed consent to treatment (or refuse it) and the harms from any potential assault, provide the ethical grounds for seclusion. This takes an empirical rights-based approach to the ethical arguments, recognised in John Stuart Mill's work, On Liberty (Mill 1869), the philosophical treatise on which much of the 
modern Western legislation surrounding freedoms is constructed. He states:

'the only purpose for which power can be rightfully exercised over any member of a civilized community, against his will, is to prevent harm to others. His own good, either physical or moral, is not a sufficient warrant.'

Both of these arguments (the minimisation of harm and presumed lack of autonomy/capacity to consent) rely on the use of seclusion only in restrictive circumstances. In many jurisdictions, seclusion can only occur when a patient is legally detained under a Mental Health Act. This legal framework protects the patient's liberties and ensures that psychiatrists are able to justify in law the grounds for seclusion. Alongside this rigid requirement, national policy ensures a clear framework under which secluded patients are monitored and reviewed, enabling the period of time in seclusion to be as brief as possible. Guidelines, such as the Code of Practice in England and Wales (Department of Health 2008), provide clarification on implementation to make application as straightforward as possible. Although the specific conditions making seclusion legal vary from country to country, the principle of use to manage behaviour for the least amount of time in the safest way possible remains. Table 1 outlines the basic legal tenets in some Western countries as an example of this.

\section{Initial assessment of prior to seclusion}

Before considering seclusion for an acutely disturbed patient, it is important to investigate

TABLE 1 Principal restrictions to the use of seclusion

\begin{tabular}{|c|c|c|c|c|}
\hline & $\begin{array}{l}\text { England } \\
\text { and Wales }\end{array}$ & Ireland ${ }^{b}$ & $\begin{array}{c}\text { New } \\
\text { Zealand }^{c}\end{array}$ & $\begin{array}{l}\text { Victoria, } \\
\text { Australia }\end{array}$ \\
\hline $\begin{array}{l}\text { As a last resort to manage the } \\
\text { patient }\end{array}$ & Yes & Yes & Yes & Yes \\
\hline For the shortest time possible & Yes & Yes & Yes & Yes \\
\hline As part of a management plan & Yes & Yes & & Yes \\
\hline Benefits outweigh risks & Yes & & Yes & \\
\hline Never as a punishment & Yes & & Yes & \\
\hline Never because of staff shortages & Yes & & Yes & \\
\hline $\begin{array}{l}\text { Never part of a routine } \\
\text { management }\end{array}$ & & & Yes & \\
\hline Must be culturally appropriate & & Yes & Yes & Yes \\
\hline $\begin{array}{l}\text { Safety of patients, visitors and } \\
\text { staff is equal }\end{array}$ & & Yes & & \\
\hline Must be detained & No & Yes & Yes & No \\
\hline
\end{tabular}

a. Department of Health (2008).

b. Mental Health Commission (2009)

c. Ministry of Health (2008).

d. Mental Health, Drugs and Regions Division (2011). what the cause of the disturbance may be. Often this is obvious in patients whose psychiatric diagnosis is clear and who are well known to the treating service. However, this may not be the case for newly presenting patients or those in whom the diagnosis is not clear. It remains important to rule out potentially treatable medical causes both acutely and during the period of in-patient care. Ensuring sufficient information is present in the patient's file to confirm the diagnosis and contacting the community team, if the patient is known, allows for confirmation of the pattern of disturbance, ensuring it is typical for the individual. If there is any suspicion of a medical cause for the patient's agitation, this requires assessment prior to or immediately following initiation of seclusion. This includes taking a brief history from the patient, staff or family, measurement of vital signs (including pulse, blood pressure, respiration rate, blood sugar and temperature) and physical examination insofar as is possible (particularly guided by history). If there are any indicators of a medical problem, a referral to the accident and emergency department for further investigation should be made. Clearly, combined care remains important in such cases and this may include pharmacotherapy for agitation if de-escalation is ineffective, and specialist mental health nursing support. As the case vignette of fictitious Ms T shows, ignoring this step can have significant detrimental consequences (Box 2).

An increasing number of patients have advanced directives in relation to their mental healthcare and if possible these requests should be read and considered. Often they will detail a patient's preferences with respect to medication, de-escalation strategies and the use of seclusion or a psychiatric intensive care unit. In most jurisdictions, if behavioural disturbance is sufficiently severe to warrant consideration of seclusion, an assessment in law and initiation of the relevant mental health act is also urgently required. This ensures that any seclusion is legal and provides the appropriate legal protections for both patient and doctor. Finally, keeping family involved in the process of assessment and initial management both allows clarity of history immediately leading to the assessment and minimises the potential for complaints about the process of the assessment in the future.

\section{Characteristics of secluded patients}

Any patient may become sufficiently disturbed to require seclusion, but it is more common in some clinical groups. The most common age range for patients who are secluded is 28-38 years (Gerlock 1983), and interventions aimed at the patient, 
B0X 2 Case vignette: Ms T

Ms T, a 36-year-old woman, was brought to the accident and emergency department (A\&E) by her husband, who said that once again he was unable to care for her and their three children at home. He explained that she had become erratic in her behaviour, was not looking after the children and made little sense when spoken to. She had stopped cooking the meals and was not cleaning the house. Occasionally she would lash out, apparently irritated for no obvious reason. He was concerned by her behaviour and worried she may, inadvertently, harm their children.

In the previous 6 months, Ms T had presented twice to A\&E with similar behaviours requiring psychiatric hospital admission. On both occasions her behaviour had been so erratic that she had been secluded for short periods of time. Both admissions lasted approximately a week before discharge home. She did not attend the follow-up appointments arranged for her in the community.
Routine physical examination on the previous two visits was unremarkable, as were routine bloods. On the basis of her history and presentation, the A\&E staff called the psychiatric crisis team, who reviewed her briefly and agreed to admission.

She was transported to the psychiatric ward, where she quickly became agitated and distressed and was secluded for her safety. She was reviewed medically after an hour of seclusion, during which time $10 \mathrm{mg}$ of olanzapine and $2 \mathrm{mg}$ of lorazepam had been given orally. Nursing staff explained that they knew the patient well, as she had been admitted twice previously, and they felt it likely she would sleep through the night until breakfast; however, as it was late, overnight seclusion was the safest course of action. When reviewed she was drowsy on the bed and did not answer questions clearly. Her husband had left to care for their children and could not be contacted. There was therefore minimal history available.
She was left asleep in the seclusion room so as not to risk another episode of aggression and the seclusion documents were signed for the night.

The following morning a brief physical exam was unremarkable other than a pulse of $110 \mathrm{bpm}$. As no physical investigations had been taken in A\&E the night before, bloods were taken for full blood count, urea and electrolytes, and thyroid function tests. A finger prick showed a blood glucose of $1.6 \mathrm{mmol} / \mathrm{l}$. She was therefore woken and given sugar. The medical team was contacted and she was transferred to a medical ward with psychiatric nursing support and referral to the liaison service. Investigation revealed an insulinoma, which was surgically removed; however, Ms T was left with permanent cognitive problems and a change in personality, being more erratic and less able to cope at home. It was considered most likely that all her presentations had been due to the developing tumour. taking age into account (e.g. patient-focused care), may reduce seclusion time (Sullivan 2004). It is worth noting that, although young patients are secluded more frequently, older patients may be secluded for longer (Smith 2005). The literature, however, is conflicted, with some papers reporting no difference in age between secluded and nonsecluded in-patients or in length of seclusion. No other demographic variable differentiates secluded patients from their non-secluded peers, with gender (e.g. Mason 1998) and ethnicity (Gudjonsson 2004) showing no clear differences.

The most predictive diagnostic indicator is schizophrenia (El-Badri 2002), followed by bipolar affective disorder and personality disorder (Stolker 2006). Comorbid substance misuse is also common.

One study of almost 1000 in-patients in The Netherlands (Stolker 2005) found a close correlation between scores on the Global Assessment of Functioning (GAF) scale and seclusion. Scores $<55$ were correlated with higher seclusion rates, and the lower the score, the stronger the correlation. This indicates that greater impairment in social functioning is associated with increased use of seclusion (Box 3). There are also single studies that have found associations with low cholesterol (Repo-Tiihonen 2002) and low prolactin levels (Nakajima 2003).

These markers (GAF, cholesterol and prolactin) are potential avenues for further investigation to enable identification of patients who are more likely to be secluded. By identifying 'at risk' patients at the point of hospital admission, individual measures to reduce the risk of seclusion could be implemented in the future. There is insufficient information currently to recommend their routine clinical use.

\section{Reasons for initiating seclusion}

Seclusion may be initiated for a number of reasons within the confines of the law, as outlined earlier, the most common reason being aggression. The majority of studies identify physical aggression as the immediate antecedent to the initiation of seclusion, although verbal aggression (threats)

\section{BOX 3 Features of secluded patients ${ }^{a}$}

\section{Characteristics found in secluded patients}

- Young adults, 28-38 years

- Diagnosis of schizophrenia, bipolar affective disorder or personality disorder

- History of alcohol and drug use

- Lower Global Assessment of Functioning score $(<55)$

No difference in secluded and non-secluded patients

- By gender

- By ethnicity

- By relationship status

a. Data in the literature are mixed, but on balance the characteristics described here are found. 
also often initiates seclusion. Some studies suggest that seclusion is initiated in direct response to the patient's psychological disorder, whereas others suggest that it is the result of their disruptive behaviour. These situations appear to be similar (and possibly are), although they are rarely more specifically defined. Other reasons for initiating seclusion include: risk of absconding, refusing to take medication as treatment, and refusing to cooperate with the in-patient team.

These reasons, although they may have some clinical utility, likely breach the United Nations' principles for the protection of persons with mental illness and the improvement of mental healthcare (United Nations 1991) and are not in line with the policy and guidelines in many Western countries. This highlights the importance of clear clinical reasoning for the use of seclusion and a good understanding of the framework within which seclusion can be initiated.

\section{Is seclusion an effective management tool?}

As a management strategy, seclusion provides clinicians, primarily ward nursing staff, a means to manage disruptive behaviour/behavioural disturbance and to ensure the smooth and safe running of the psychiatric ward. Whether seclusion should be thought of as a treatment is controversial, as is whether it provides any specific benefits for the patient.

A Cochrane review (Sailas 2000) of seclusion and restraint was unable to find any controlled trials of seclusion in the 2155 citations found by literature review and could not therefore find any evidence-based support for the benefits of secluding patients. The authors note that, 'There are reports of serious adverse effects for these techniques in qualitative reviews'. A Cochrane review of containment strategies in psychiatric practice was also negative and could not find evidence to support their use (Muralidharan 2006). Both reviews caution against the use of seclusion on the basis of a lack of evidence.

Multiple qualitative studies explore the patient's perspective of seclusion. These studies largely identify negative affect associated with seclusion, with patients feeling angry, sad and hopeless at being secluded. Many felt coerced and expressed the view that the management of their behavioural disturbance could have been different. A minority of patients report retrospectively the positive experience of feeling 'looked after', although this was a secondary theme. Juxtaposing this, many staff felt that seclusion was beneficial to patients and to the overall experience of being an in-patient (Newton-Howes 2011).
Intervention studies on seclusion aim to reduce the rates of seclusion as their primary outcome, examining whether interventions to reduce the rates are effective, rather than examining whether seclusion itself leads to any improvement (or deterioration) in overall patient management. These studies all assume that seclusion is detrimental and that its use therefore needs to be reduced. Consequently, it is difficult to identify any clear evidence from trials that seclusion is beneficial and this needs to be borne in mind when considering seclusion and its use. Other data provide insights into the potential effects of secluding patients, although the methodological weaknesses of retrospective and case-review studies limit the conclusions that can be drawn.

A study in an Australian forensic setting (Daffen 2003) indicated that levels of aggression did not predict between secluded and non-secluded patients, suggesting that seclusion did not reduce further episodes of aggression nor reduced seclusion rates. A study from the USA explored the correlation between seclusion and length of hospital admission and found a positive correlation between them (Gerlock 1983). Although directionality cannot be assumed, one interpretation of this study would be that secluding patients potentially lengthens their hospital admission, a proxy measure commonly used to measure illness severity. More recently, a study from New Zealand found that prolonged seclusion times were correlated with poorer treatment (Tyrer 2012). The authors speculate about the cultural reasons for this, suggesting that more 'appropriate treatment' when secluded would minimise seclusion times and admission to hospital. These types of studies highlight potentially negative correlations between seclusion and outcome.

\section{Strategies that may minimise the use of seclusion}

Given the relative lack of evidence for the benefits of seclusion, trials of interventions intended to minimise its use have been conducted. This strategy reflects the social policy of most countries in gradually improving patient autonomy and choice in their own recovery. The interventions are often complex and multifaceted, making interpretation of results difficult. In addition, some trials showed significant increases in seclusion rates and no trial has been able to reliably pinpoint the key component(s) that effect positive change. This literature has been reviewed by Gaskin et al (2007), who identified 17 interventions that may lead to reductions in seclusion rates. These fall into three broad spheres: interventions at a policy level, 
interventions at a hospital level and interventions at an individual (staff) level.

\section{Policy- and hospital-level interventions}

Policy-level interventions involve increases in state support for psychiatric hospitals, and policy and regulation designed to closely monitor the use of seclusion. Hospital-based interventions are described more broadly. They include improvements in leadership (within both clinical and management arms), close monitoring, audit, examination of the process of seclusion for individual patients and integration between staff and management, as described in Box 4. These changes are designed to identify and reduce systematic problems in the use of seclusion. Clear management plans for each patient, with an emphasis on behavioural interventions and improvements in monitoring patients' episodes in the seclusion setting, are hospital-based policies designed to ensure that all other options are considered prior to seclusion, and that patients leave seclusion as soon as practicable. Finally, improving the hospital setting itself in terms of the physical and psychological environment, and clearly positioning hospital-based care within

BOX 4 Interventions that may reduce seclusion rates

Policy-level interventions

- State support

- State policy and regulation

Hospital-level interventions

- Leadership

- Examination

- Integration of clinical and management teams

- Treatment planning

- Monitoring seclusion episodes

- Changing the therapeutic environment

- Changing the physical environment

- Having a hospital-wide focus

Individual-level interventions

- Increasing staff:patient ratios

- Psychiatric emergency teams

- Staff education

- Improved seclusion monitoring

- Pharmacotherapy

- Patients as participants in planning

- Improving staff welfare

(Based on the categories identified by Gaskin 2007) the wider service, shows benefits in terms of minimising seclusion time within services.

\section{Individual-level interventions}

On an individual staff level, a number of interventions are identified that are beneficial in reducing seclusion rates:

- improving staff:patient ratios

- ensuring that education about the policies, ethical and legal requirements of seclusion occurs regularly

- educating staff about methods to manage disturbance without needing to seclude patients

- improving individual patient monitoring while secluded

- involving patients as active decision makers.

As many of these interventions occur in a multifaceted way in the trials identified, it is not possible to rank them by importance or effectiveness, although all have some evidence of benefit. It is, however, worth noting that the literature is too conflicting to provide clear evidence-based alterations with any certainty. The most common themes in these papers associated with reductions in seclusion rates are improved clinical-management communication, improving staffing levels and improvements in the physical environment (Box 4).

\section{Conclusions}

Despite the potential legal, ethical and clinical challenges associated with seclusion, it is likely that it will remain a part of psychiatric in-patient care for the foreseeable future. It is increasingly recognised as a necessary intervention to maintain individual patient safety and ensure the safe and therapeutic management of a psychiatric in-patient ward. However, there is a dearth of clear evidence as to its benefit associated with the peripheral evidence of harm, emphasising the importance of using seclusion both carefully and thoughtfully. The increasing regulation surrounding seclusion recognises this, and there is clearly a need for further robust studies to continuously improve the use of seclusion and minimise any harms associated with it.

\section{Acknowledgement}

I would like to thank Dr MacDonald for her helpful review and comments on the initial draft of this paper.

\section{References}

Daffen M, Olgoff J, Howells K (2003) Agression in an Australian forensic psychiatric hospital. British Journal of Forensic Practice 5: 18-28. 
Davidson L (2005) Recovery, self management and the expert patient changing the culture of mental health from a UK perspective. Journal of Mental Health 14: 25-35.

Department of Health (2008) Code of Practice: Mental Health Act 1983. TSO (The Stationery Office)

El-Badri S, Mellsop G (2002) A study of the use of seclusion in an acute psychiatric service. Australian and New Zealand Journal of Psychiatry 36: 399-403

Gaskin CJ, Elsom SJ, Happell B (2007) Interventions for reducing the use of seclusion in psychiatric facilities. Review of the literature. British Journal of Psychiatry 191: 298-303.

Gerlock A, Solomons H (1983) Factors associated with the seclusion of psychiatric patients. Perspectives in Psychiatric Care 21: 59-64

Gudjonsson GH, Rabe-Hesketh S, Szmukler G (2004) Management of psychiatric in-pateint violence: patient ethnicity and use of medication, restraint and seclusion. British Journal of Psychiatry 184: 258-62.

Holmes D (2004) The mentally ill and social exclusion: a critical examination of the use of seclusion from the patient's perspective. Issues in Mental Health Nursing 25: 559-78.

Knox D, Holloman G (2012) Use and avoidance of seclusion and restraint: Consensus Statement of the American Association for Emergency Psychiatry Project BETA Seclusion and Restraint Working Group. Western Journal of Emergency Medicine 13: 35-9.

Mason T (1994) Seclusion: an international comparison. Medicine, Science and the Law 34: 54-60.

Mason T (1998) Gender differences in the use of seclusion. Medicine, Science and the Law 38: 2-9.

Mental Health Commission (2009) Rules Governing the Use of Seclusion and Mechanical means of Bodily Restraint - Version 2. Mental Health Commission.

Mental Health, Drugs \& Regions Division (2011) Seclusion in Approved Mental Health Services (Chief Psychiatrist's Guideline). Victorian Government Department of Health.

Mill JS (1869) On Liberty. Available at: http://www.gutenberg.org/ files/34901/34901-h/34901-h.htm

Ministry of Health (2008) Health and Disability Services (Restraint Minimisation and Safe Practice) Standards (NZS 8134.2:2008). Standards Council.
Morrall P, Muir-Cochrane E (2002) Naked social control: seclusion and psychiatric nursing in post-liberal society. Australian e-Journal for the Advancement of Mental Health 1: 1-12.

Muralidharan S, Fenton M (2006) Containment strategies for people with serious mental illness. Cochrane Database of Systematic Reviews 3: CD002084.

Nakajima J, Terao T, Nakamura J (2003) Characteristics of repeated secluded elderly female schizophrenic in-patients. Progress in NeuroPsychopharmacology and Biological Psychiatry 25: 771-4.

Newton-Howes G, Mullen R (2011) Coercion in psychiatric care: systematic review of correlated and themes. Psychiatric Services 62: 465-70.

Public Policy Committee (1990) The seclusion of psychiatric patients. Psychiatric Bulletin 14: 754-6.

Repo-Tiihonen E, Paavola P, Halonen P, et al (2002) Seclusion treatment measures and serum cholesterol levels among Finnish male forensic psychiatric patients. Journal of Forensic Psychiatry 13: 157-65.

Sailas E, Fenton M (2000) Seclusion and restraint for people with serious mental illness. Cochrane Database of Systematic Reviews 1: CD001163.

Smith G, Davis R, Bixler E, et al (2005) Pennsylvania state hospital systems's seclusion and restraint reduction program. Psychiatric Services 56: 1115-22.

Sowers W (2005) Transforming systems of care: the American Association of Community Psychiatrists guidelines for recovery oriented services. Community Mental Health Journal 41: 757-74.

Stolker J, Hugenholtz G, Heerdink E (2005) Seclusion and the use of antipsychotics in hospitalized psychiatric patients. Psychology, Crime and Law 11: 489-95.

Stolker J, Nijman H, Zwanikken P-H (2006) Are patients' views on seclusion associated with lack of privacy on the ward? Archives of Psychiatric Nursing 20: 282-7.

Sullivan D. Wallis M, Lloyd C (2004) Effects of patient-focused care on seclusion in a psychiatric intensive care unit. International Journal of Therapy and Rehabilitation 11: 503-8.

Tyrer S, Beckley J, Goel D, et al (2012) Factors affecting the practice of seclusion in an acute mental health service in Southland, New Zealand. Psychiatrist 36: 214-8.

United Nations (1991) The Protection of Persons with Mental IIIness and the Improvement of Mental Health Care. UN (http://www.un.org/ documents/ga/res/46/a46r119.htm)

\section{MCOs}

Select the single best option for each question stem

1 The following are key descriptors of seclusion:

a the patient is isolated from others

b seclusion is an integral part of the treatment plan

c the patient is in a room they can leave

$\mathrm{d}$ there is no furniture in the room

e the patient is observed intermittently.

2 The primary restrictions to seclusion include:

a its use to enable adequate pharmacotherapy

$\mathrm{b}$ its use to manage staff shortages

c the use of routine times in seclusion $\mathrm{d}$ the patient must be detained under the Menta Health Act

e ensuring it is the best first response to manage disturbance.

3 The common characteristics of secluded patients include:

a aged between 55 and 64

b male

c intoxicated

d low GAF score

e belonging to an ethnic minority.

4 Seclusion has been proved to be valuable in:

a shortening hospital admissions

b anecdotal descriptions of management of aggression c improving the patient's experience of in-patient care

d reducing coercion

e improving symptoms.

\section{Strategies that have evidence showing} they reduce seclusion rates include:

a devolution away from state regulation

b improved staff:patient ratios

c minimal decoration in hospital wards

$\mathrm{d}$ having management leave policy to clinical staff

e improving the welfare of ward management. 